\title{
On the Exact Maximum Complexity of Minkowski Sums of Polytopes
}

\author{
Efi Fogel · Dan Halperin · Christophe Weibel
}

Received: 23 November 2007 / Revised: 29 December 2008 / Accepted: 16 March 2009 /

Published online: 8 April 2009

(C) Springer Science+Business Media, LLC 2009

\begin{abstract}
We present a tight bound on the exact maximum complexity of Minkowski sums of polytopes in $\mathbb{R}^{3}$. In particular, we prove that the maximum number of facets of the Minkowski sum of $k$ polytopes with $m_{1}, m_{2}, \ldots, m_{k}$ facets, respectively, is bounded from above by $\sum_{1 \leq i<j \leq k}\left(2 m_{i}-5\right)\left(2 m_{j}-5\right)+\sum_{1 \leq i \leq k} m_{i}+\left(\begin{array}{l}k \\ 2\end{array}\right)$. Given $k$ positive integers $m_{1}, m_{2}, \ldots, m_{k}$, we describe how to construct $k$ polytopes with corresponding number of facets, such that the number of facets of their Minkowski sum is exactly $\sum_{1 \leq i<j \leq k}\left(2 m_{i}-5\right)\left(2 m_{j}-5\right)+\sum_{1 \leq i \leq k} m_{i}+\left(\begin{array}{l}k \\ 2\end{array}\right)$. When $k=2$, for example, the expression above reduces to $4 m_{1} m_{2}-9 m_{1}-9 m_{2}+26$.
\end{abstract}

Keywords Polyhedra · Minkowski sum · Gaussian maps · Complexity

This work has been supported in part by the IST Programme of the EU as Shared-cost RTD (FET Open) Project under Contract No IST-006413 (ACS-Algorithms for Complex Shapes), by the Israel Science Foundation (grant no. 236/06), and by the Hermann Minkowski-Minerva Center for Geometry at Tel Aviv University.

A preliminary version appeared in Proc. 23rd ACM Symposium on Computational Geometry, pp. 319-326, Gyeongju, South Korea, 2007.

E. Fogel $(\bowtie) \cdot$ D. Halperin

School of Computer Science, Tel-Aviv University, Tel-Aviv 69978, Israel

e-mail: efif@post.tau.ac.il

D. Halperin

e-mail: danha@post.tau.ac.il

C. Weibel

Department of Mathematics, McGill University, Montreal, Canada

e-mail: weibel@math.mcgill.ca 


\section{Introduction}

Let $P$ and $Q$ be two compact convex polyhedra in $\mathbb{R}^{d}$. The Minkowski sum of $P$ and $Q$ is the convex polyhedron, polytope for short, $M=P \oplus Q=\{p+q \mid p \in P$, $q \in Q\}$.

Minkowski-sum computation constitutes a fundamental task in computational geometry. Minkowski sums are frequently used in areas such as robotics and motion planing $[13,17]$ and many additional domains like solid modeling, design automation, manufacturing, assembly planning, virtual prototyping, etc., as Minkowski sums are closely related to proximity queries [16]. For example, the minimum translation separation-distance between two objects $P$ and $Q$ is equal to the minimum distance between the origin and the boundary of $P \oplus(-Q)$, where $-Q$ is the reflection of $Q$ through the origin [5]. Minkowski sums are also primary operations in some techniques that morph three-dimensional objects [15].

The combinatorial complexity of the Minkowski sum of two polygons of $m$ and $n$ vertices, respectively, is $\Theta\left(m^{2} n^{2}\right)$ (see, e.g., [1]). The corresponding tight bound on the complexity of Minkowski sum of two polyhedra in $\mathbb{R}^{3}$ with $m$ and $n$ vertices, respectively, is $\Theta\left(m^{3} n^{3}\right)$. Various methods to compute the Minkowski sum of two polyhedra in $\mathbb{R}^{3}$ have been proposed. One common approach is to decompose each polyhedron into convex pieces, compute pairwise Minkowski sums of pieces of the two, and finally the union of the pairwise sums. Computing the Minkowski sum of two convex polyhedra remains a key operation. The combinatorial complexity of the sum can be as high as $\Theta(m n)$ when both polyhedra are convex. For the complexity of the intermediate case, where only one polyhedron is convex, cf. [2, 17].

\subsection{Minkowski Sums of Polytopes in $\mathbb{R}^{3}$}

One method to compute the Minkowski sum of two polytopes is to compute the convex hull of the pairwise sum of the vertices of the two polytopes. While being simple and easy to implement, the time complexity of this method is $\Omega(\mathrm{mn})$ regardless of the size of the resulting sum, which can be as low as $(m+n)$ (counting facets) for degenerate cases. ${ }^{1}$ Several output sensitive algorithms to compute Minkowski sums of polytopes in $\mathbb{R}^{3}$ have been proposed. Basch et al. [3] extended the concept of convolution introduced by Guibas et al. [12] and presented an algorithm to compute the convolution in three dimensions. Gritzmann and Sturmfels [11] obtained a polynomial-time algorithm in the input and output sizes for computing Minkowski sums of $k$ polytopes in $\mathbb{R}^{d}$ for a fixed dimension $d$, and Fukuda [7] provided an output-sensitive polynomial-time algorithm for variables $d$ and $k$. Ghosh [9] presented a unified algorithm for computing 2D and 3D Minkowski sums of both convex and non-convex polyhedra based on a slope diagram representation. Bekker and Roerdink [4] provided a few variations on the same idea.

Recently a few complete implementations of methods for computing exact Minkowski sums (beyond the naive method mentioned above) have need introduced:

\footnotetext{
${ }^{1}$ It can be as low as $m(=n)$ in the extremely-degenerate case of two similar polytopes with parallel facets.
} 
(i) a method based on Nef polyhedra embedded on the sphere [10], (ii) an implementation of Fukuda's algorithm by Weibel [18], and (iii) a method based on the cubical Gaussian-map data structure [6]. These methods exploit efficient innovative techniques in the area of exact geometric computing to minimize the time it takes to ensure exact results. However, even with the use of these techniques, the amortized time of a single arithmetic operation is larger than the time it takes to carry out a single arithmetic operation on native number types, such as floating point. Thus, the constant that scales the dominant element in the expression of the time complexity of these algorithms increases, which makes the question this paper attempts to answer, "What is the exact maximum complexity of Minkowski sums of polytopes in $\mathbb{R}^{3}$ ?", even more relevant.

Gritzmann and Sturmfels [11] also formulated an upper bound on the number of features $f_{i}^{d}$ of any given dimension $i$ of the Minkowski sum of many polytopes in $d$ dimensions: $f_{i}^{d}\left(P_{1} \oplus P_{2} \oplus \cdots \oplus P_{k}\right) \leq 2\left(\begin{array}{l}j \\ i\end{array}\right) \sum_{h=0}^{d-i-1}\left(\begin{array}{c}j-i-1 \\ h\end{array}\right)$ for $i=0,1, \ldots, d-1$, where $j$ denotes the number of non-parallel edges of $P_{1}, P_{2}, \ldots, P_{k}$. According to this expression, the number of facets $f_{2}^{3}$ of the Minkowski sum of two polytopes in $\mathbb{R}^{3}$ is bounded from above by $j(j-1)$. Fukuda and Weibel [8] obtained upper bounds on the number of edges and facets of the Minkowski sum of two polytopes in $\mathbb{R}^{3}$ in terms of the number of vertices of the summands: $f_{2}^{3}\left(P_{1} \oplus P_{2}\right) \leq f_{0}^{3}\left(P_{1}\right) f_{0}^{3}\left(P_{2}\right)+$ $f_{0}^{3}\left(P_{1}\right)+f_{0}^{3}\left(P_{2}\right)-6$. They also studied the properties of Minkowski sums of perfectly centered polytopes and their polars, and provided a tight bound on the number of vertices of the sum of polytopes in any given dimension.

The main result of the paper follows.

Theorem 1 Let $P_{1}, P_{2}, \ldots, P_{k}$ be a set of $k$ polytopes in $\mathbb{R}^{3}$, such that the number of facets of $P_{i}$ is $m_{i}$ for $i=1,2, \ldots, k$. The maximum number of facets of the Minkowski sum $P_{1} \oplus P_{2} \oplus \cdots \oplus P_{k}$ is $\sum_{1 \leq i<j \leq k}\left(2 m_{i}-5\right)\left(2 m_{j}-5\right)+\sum_{i=1}^{k} m_{i}+\left(\begin{array}{l}k \\ 2\end{array}\right)$. This bound is tight. Namely, given $k$ positive integers $m_{1}, m_{2}, \ldots, m_{k}$, it is possible to construct $k$ polytopes with corresponding number of facets, such that the number of facets of their Minkowski sum is exactly the expression above.

In Sects. 2 and 3, we prove the special case $k=2$, where the expression for the bound reduces to $4 m_{1} m_{2}-9 m_{1}-9 m_{2}+26$. We prove the general case in Sect. 4 . Snapshots of several pre-constructed polytopes, the Minkowski sum of which is maximal, are available at http://www.cs.tau.ac.il/ efif/Mink. The polyhedra models and an interactive program that computes their Minkowski sums and visualizes them can be downloaded as well.

\section{The Upper Bound for $k=2$}

The Gaussian Map $G=G(P)$ of a compact convex polyhedron $P$ in Euclidean threedimensional space $\mathbb{R}^{3}$ is a set-valued function from $P$ to the unit sphere $\mathbb{S}^{2}$, which assigns to each point $p$ the set of outward unit normals to support planes to $P$ at $p$. Thus, the whole of a facet $f$ of $P$ is mapped under $G$ to a single point, representing the outward unit normal to $f$. An edge $e$ of $P$ is mapped to a (geodesic) segment $G(e)$ 


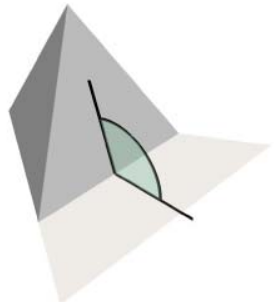

(a)

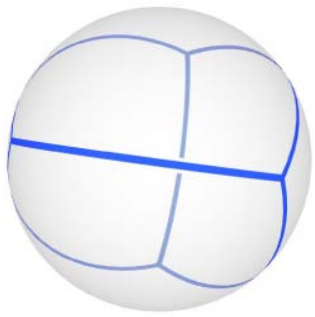

(b)

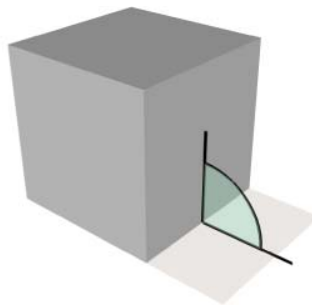

(c)

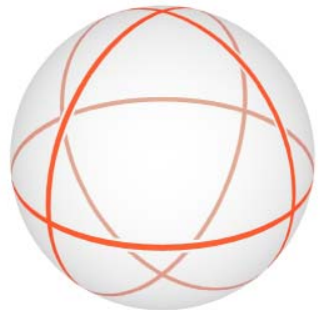

(d)

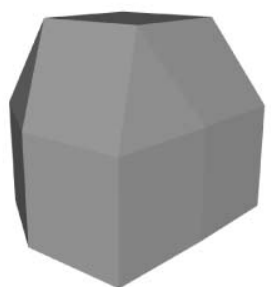

(e)

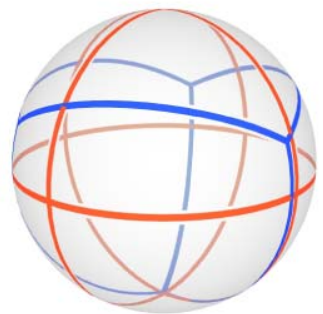

(f)

Fig. 1 a A tetrahedron, b the Gaussian map of the tetrahedron, $\mathbf{c}$ a cube, $\mathbf{d}$ the Gaussian map of the cube, $\mathbf{e}$ the Minkowski sum of the tetrahedron and the cube, and $\mathbf{b}$ the Gaussian map of the Minkowski sum

on $\mathbb{S}^{2}$, whose length is easily seen to be the exterior-dihedral angle at $e$. A vertex $v$ of $P$ is mapped by $G$ to a spherical polygon $G(v)$, whose sides are the images under $G$ of edges incident to $v$, and whose angles are the angles supplementary to the planar angles of the facets incident to $v$; that is, $G\left(e_{1}\right)$ and $G\left(e_{2}\right)$ meet at angle $\pi-\alpha$ whenever $e_{1}$ and $e_{2}$ meet at angle $\alpha$ [14].

The overlay of two planar subdivisions $\mathcal{S}_{1}$ and $\mathcal{S}_{2}$ is a planar subdivision $\mathcal{S}$, such that there is a face $f$ in $\mathcal{S}$ if and only if there are faces $f_{1}$ and $f_{2}$ in $\mathcal{S}_{1}$ and $\mathcal{S}_{2}$, respectively, such that $f$ is a maximal connected subset of $f_{1} \cap f_{2}$. The overlay of two (two-dimensional) subdivisions embedded on a sphere is defined similarly. The overlay of the Gaussian maps of two polytopes $P$ and $Q$ respectively identifies all pairs of features of $P$ and $Q$ that have parallel supporting planes, as they occupy the same space on the unit sphere, thus, identifying all the pairwise features that contribute to the boundary of the Minkowski sum of $P$ and $Q$. A facet of the Minkowski sum is either a facet $f$ of $Q$ translated by a vertex of $P$ supported by a plane parallel to $f$, or vice versa, or it is a facet parallel to the two parallel planes supporting an edge of $P$ and an edge of $Q$ respectively. A vertex of the Minkowski sum is the sum of two vertices of $P$ and $Q$ respectively supported by parallel planes.

The number of facets of the Minkowski sum $M$ of two polytopes $P$ and $Q$ with $m$ and $n$ facets respectively is equal to the number of vertices of the Gaussian map $G(M)$ of $M$. A vertex in $G(M)$ is either due to a vertex in the Gaussian map of $P$, or due to a vertex in the Gaussian map of $Q$, or due to an intersection of exactly two edges, one of the Gaussian map of $P$ and the other of the Gaussian map of $Q$. Thus, 
the number of facets of $M$ cannot exceed $m+n+g(M)$, where $g(M)$ is the number of intersections of edges in $G(M){ }^{2}$

Observation 2 The maximum exact number of edges in a Gaussian map $G(P)$ of a polytope $P$ with $m$ facets is $3 m-6$. The maximum exact number of faces is $2 m-4$. Both maxima occur at the same Gaussian maps.

The above can be obtained by a simple application of Euler's formula for planar graphs to the Gaussian map $G(P)$. It can be used to trivially bound the exact maximum number of facets of the Minkowski sum of two polytopes defined as $f(m, n)=\max \{f(P \oplus Q) \mid f(P)=m, f(Q)=n\}$, where $f(P)$ is the number of facets of a polytope $P$. First, we can use the bound on the number of edges to obtain: $f(m, n) \leq m+n+(3 m-6) \cdot(3 n-6)=9 m n-17 m-17 n+36$. Better yet, we can plug the bound on the number of dual faces, which is the number of primal vertices, in the expression introduced by Fukuda and Weibel, see Sect. 1.1, to obtain: $f(m, n) \leq(2 m-4) \cdot(2 n-4)+(2 m-4)+(2 n-4)-6=4 m n-6 m-6 n+2$. Still, we can improve the bound even further, but first we need to bound the number of faces in $G(M)$.

Lemma 3 Let $G_{1}$ and $G_{2}$ be two Gaussian maps of convex polytopes, and let $G$ be their overlay. Let $f_{1}, f_{2}$, and $f$ denote the number of faces of $G_{1}, G_{2}$, and $G$, respectively. Then, $f \leq f_{1} \cdot f_{2}$.

Each face in the overlay is an intersection of a face of each map. Since these faces are spherically convex (and smaller than hemispheres), the intersection is also spherically convex (and thus connected).

We are ready to tackle the upper bound of Theorem 1 for the special case $k=2$, that is, prove that the number of facets of the Minkowski sum $P \oplus Q$ of two polytopes $P$ and $Q$ with $m$ and $n$ facets, respectively, cannot exceed $4 m n-9 m-9 n+26$.

Proof Let $v_{1}, e_{1}, f_{1}$ and $v_{2}, e_{2}, f_{2}$ denote the number of vertices, edges, and faces of $G(P)$ and $G(Q)$, respectively. The number of vertices, edges, and faces of $G(M)$ is denoted as $v, e$, and $f$, respectively. Assume that $P$ and $Q$ are two polytopes such that the number of facets of their Minkowski sum is maximal. Recall that the number of facets of a polytope is equal to the number of vertices of its Gaussian map. Thus, we have $v_{1}=m, v_{2}=n$, and $v=f(m, n)$. First, we need to show that vertices of $G(P)$, vertices of $G(Q)$, and intersections between edges of $G(P)$ and edges of $G(Q)$ do not coincide. Assume to the contrary that some do. Then, one of the polytopes $P$ or $Q$ or both can be slightly rotated to escape this degeneracy, but this would increase the number of vertices $v=f(m, n)$, contradicting the fact that $f(m, n)$ is maximal. Therefore, the number of vertices $v$ is exactly equal to $v_{1}+v_{2}+v_{x}$, where $v_{x}$ denotes the number of intersections of edges of $G(P)$ and edges of $G(Q)$ in $G(M)$. Counting the degrees of all vertices in $G(M)$ implies that $2 e_{1}+2 e_{2}+4 v_{x}=2 e$. Using Euler's

\footnotetext{
${ }^{2}$ The number of facets is strictly equal to the given expression only when no degeneracies occur.
} 
formula, we get $e_{1}+e_{2}+2 v_{x}=f+v_{1}+v_{2}+v_{x}-2$. Applying Lemma 3, we can bound $v_{x}$ from above $v_{x} \leq f_{1} f_{2}+v_{1}+v_{2}-2-e_{1}-e_{2}$.

Observation 2 sets an upper bound on the number of edges $e_{1}$. Thus, $e_{1}$ can be expressed in terms of $\ell_{1}$, a non-negative integer, as follows: $e_{1}=3 v_{1}-6-\ell_{1}$. Applying Euler's formula, the number of facets can be expressed in terms of $\ell_{1}$ as well: $f_{1}=e_{1}+2-v_{1}=2 v_{1}-4-\ell_{1}$. Similarly, we have $e_{2}=3 v_{2}-6-\ell_{2}$ and $f_{2}=2 v_{2}-4-\ell_{2}$ for some non-negative integer $\ell_{2}$.

$$
\begin{aligned}
v_{x} & \leq\left(2 v_{1}-4-\ell_{1}\right)\left(2 v_{2}-4-\ell_{2}\right)+v_{1}+v_{2}-2-\left(3 v_{1}-6-\ell_{1}\right)-\left(3 v_{2}-6-\ell_{2}\right) \\
& \leq 4 v_{1} v_{2}-10 v_{1}-10 v_{2}+26+h\left(\ell_{1}, \ell_{2}\right),
\end{aligned}
$$

where $h\left(\ell_{1}, \ell_{2}\right)=\ell_{1} \ell_{2}+5 \ell_{1}+5 \ell_{2}-2 v_{1} \ell_{2}-2 v_{2} \ell_{1}$.

$G(P)$ consists of a single connected component. Therefore, the number of edges $e_{1}$ must be at least $v_{1}-1$. This is used to obtain an upper bound on $\ell_{1}$ as follows: $v_{1}-1 \leq e_{1}=3 v_{1}-6-\ell_{1}$, which implies $\ell_{1} \leq 2 v_{1}-5$, and similarly $\ell_{2} \leq 2 v_{2}-5$. Thus, we have:

$$
\begin{aligned}
h\left(\ell_{1}, \ell_{2}\right) & =\ell_{1} \ell_{2}+5 \ell_{1}+5 \ell_{2}-2 v_{1} \ell_{2}-2 v_{2} \ell_{1} \\
& =\ell_{1}\left(\frac{\ell_{2}}{2}-\left(2 v_{2}-5\right)\right)+\ell_{2}\left(\frac{\ell_{1}}{2}-\left(2 v_{1}-5\right)\right) \leq 0 .
\end{aligned}
$$

From (1), we get that $v_{x} \leq 4 v_{1} v_{2}-10 v_{1}-10 v_{2}+26$, and since $f(m, n)=v_{1}+$ $v_{2}+v_{x}$, we conclude that $f(m, n) \leq 4 v_{1} v_{2}-9 v_{1}-9 v_{2}+26$. The maximum number of facets can be reached when $h\left(\ell_{1}, \ell_{2}\right)$ vanishes. This occurs when $\ell_{1}=\ell_{2}=0$. That is, when the number of edges of $G(P)$ and $G(Q)$ is maximal. This concludes the proof of the upper bound of Theorem 1 for the special case $k=2$.

Corollary 4 The maximum number of facets can be attained only when the number of edges of each of $P$ and $Q$ is maximal for the given number of facets.

\section{The Lower Bound for $k=2$}

Given two integers $m \geq 4$ and $n \geq 4$, we describe how to construct two polytopes in $\mathbb{R}^{3}$ with $m$ and $n$ facets, respectively, such that the number of facets of their Minkowski sum is exactly $4 m n-9 m-9 n+26$, establishing the lower bound of Theorem 1 for the special case $k=2$. More precisely, given $i$, we describe how to construct a skeleton of a polytope $P_{i}$ with $i$ facets, $3 i-6$ edges, and $2 i-4$ vertices, and prove that the number of facets of the Minkowski sum of $P_{m}$ and $P_{n}$, properly adjusted and oriented, is exactly $4 m n-9 m-9 n+26$. As in the previous sections, we mainly operate in the dual space of Gaussian maps. However, the construction of the desired Gaussian maps described below is an involved task since not every arrangement of arcs of great circles embedded on the unit sphere the faces of which 
are convex and the edges of which are strictly less than $\pi$ long constitutes a valid Gaussian map.

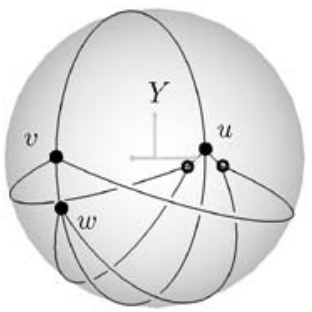

The Gaussian map of $P_{5}$

We defer the treatment of the special case $i=4$ to the sequel, and start with the general case $i \geq 5$. The figure above depicts the Gaussian map of $P_{5}$. We use the subscript letter $i$ in all notations $X_{i}$ to identify some object $X$ with the polytope $P_{i}$. For example, we give the Gaussian map $G\left(P_{i}\right)$ of $P_{i}$ a shorter notation $G_{i}$, but in this paragraph we omit the subscript letter in all notations for clarity. First, we examine the structure of the Gaussian map $G$ of $P$ to better understand the structure of $P$. Let $V$ denote the set of vertices of $G$. Recall that the number of vertices, edges, and faces of $G$ is $i, 3 i-6$, and $2 i-4$, respectively. The unit sphere, where $G$ is embedded on, is divided by the plane $y=0$ into two hemispheres $H^{-} \subset\{(x, y, z) \mid y \leq 0\}$ and $H^{+} \subset\{(x, y, z) \mid y>0\}$. Three vertices, namely $u, v$, and $w$, lie in the plane $x=0$. $u$ is located very close to the pole $(0,0,-1)$. It is the only vertex (out of the $i$ vertices) that lies in $H^{+} . v$ is located exactly at the opposite pole $(0,0,1)$, and $w$ lies in $H^{-}$very close to $v$. None of the remaining $i-3$ vertices in $V \backslash\{u, v, w\}$ lie in the plane $x=0$; they are all concentrated near the pole $(0,0,-1)$ and lie in $H^{-}$. The edge $\overline{u v}$, which is contained in the plane $x=0$, is the only edge whose interior is entirely contained in $H^{+}$. Every vertex in $V \backslash\{u, v, w\}$ is connected by two edges to $v$ and $w$, respectively. These edges together with the edge $\overline{u w}$, contained in the plane $x=0$, form a set of $2 i-5$ edges, denoted as $E^{\prime}$. The length of each of the edges in $E^{\prime}$ is almost $\pi$, due to the near proximity of $u, v$, and $w$ to the respective poles.

It is easy to verify that if the polytope $P$ is not degenerate, namely, its affine hull is 3 -space, then any edge of $G(P)$ is strictly less than $\pi$ long. Bearing this in mind, the main difficulty in arriving at a tight-bound construction is to force sufficient edges of the Gaussian map of one polytope to intersect sufficient edges of the Gaussian map of the other polytope, and on top of that force a pair of additional edges, one from each Gaussian map, to intersect as well. As shown below, this is the best one can do in terms of intersections.

The number of facets of the Minkowski sum of $P_{m}$ and $P_{n}$ is maximal, when the number of vertices in the overlay of $G_{m}$ and $G_{n}$ is maximal. This occurs, for example, when $G_{m}$ and $G_{n}$ are orthogonal, and one of them is rotated $90^{\circ}$ about the $Y$ axis, as depicted in Fig. 2 for the case of $m=n=5$. In this configuration, all the $2 m-5$ edges in $E_{m}^{\prime}$ intersect all the $2 n-5$ edges in $E_{n}^{\prime}$. All intersections occur in $H^{-}$. In addition, the edge $\overline{u v}_{m}$ intersects the edge $\overline{u v}_{n}$. The intersection point lies in $H^{+}$exactly at the pole $(0,1,0)$. Counting all these intersections results 
Fig. 2 The overlay of $G_{5}$ and $G_{5}^{\prime}$, where $G_{5}^{\prime}$ is $G_{5}$ rotated $90^{\circ}$ about the $Y$ axis

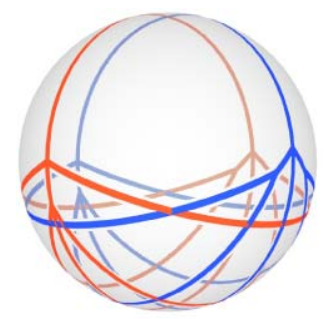

with $(2 m-5)(2 n-5)+1=4 m n-10 m-10 n+26$. Adding the original vertices of $G\left(P_{m}\right)$ and $G\left(P_{n}\right)$, yields the desired result.

Next, we explain how $P_{i}, i \geq 5$, is constructed to match the description of $G_{i}$ above. The construction of $P_{i}$ is guided by a cylinder. All the vertices of $P_{i}$ lie on the boundary of a cylinder the axis of which coincides with the $Z$ axis. We start with the case $i=5$, and show how to generalize the construction for $i>5$. The special case $i=4$ is explained last.

\subsection{Constructing $P_{5}$}

Figure 3 shows various views of $P_{5}$. Recall that $P_{5}$ has 6 vertices, denoted as $v_{0}$, $v_{1}, \ldots, v_{5}$, and 9 edges. We omit the subscript digit 5 in all the notations through the rest of this subsection for clarity. Let $\overline{v_{1} v_{2} \cdots v_{n}}$ denote the face defined by the sequence of vertices $v_{1}, v_{2}, \ldots, v_{n}$ on the face boundary. The projection of all vertices onto the plane $z=0$ lie on the unit circle. As a matter of fact, the entire face $f^{v}=$ $\overline{v_{0} v_{1} v_{2} v_{3}}$ lies in the plane $z=0$. It is mapped under $G$ to the vertex $v=G\left(f^{v}\right)$. Similarly, the faces $f^{u}=\overline{v_{5} v_{4} v_{2} v_{1}}$ and $f^{w}=\overline{v_{3} v_{4} v_{5} v_{0}}$ are mapped under $G$ to the vertices $u=G\left(f^{u}\right)$ and $w=G\left(f^{w}\right)$, respectively. Consider the projection of the vertices onto the plane $z=0$ best seen in Fig. 3b. Once the projection $v_{5}^{\prime}$ of $v_{5}$ is determined as explained below, $v_{0}$ is placed exactly on the bisector of $\angle v_{5}^{\prime} o v_{1}$. The vertices $v_{4}, v_{3}$, and $v_{2}$ are the reflection of the vertices $v_{5}, v_{0}$, and $v_{1}$, respectively, through the plane $x=0$.

Two parameters govern the exact placement of $v_{5}$ (and $v_{4}$ ). One is the size of the exterior-dihedral angle at the edge $\overline{v_{0} v_{3}}$, denoted as $\alpha$, that is, the length of the geodesic-segment that is the mapping of the edge $\overline{v w}$ of $G$. This angle is best seen in Fig. 3c. Notice, that the $Z$ axis is scaled up for clarity, and the angle in practice is much smaller. The other parameter is the size of the angle $\beta=\angle v_{4}^{\prime} o v_{5}^{\prime}$, where $v_{4}^{\prime}$ and $v_{5}^{\prime}$ are the projections of $v_{4}$ and $v_{5}$, respectively, onto the plane $z=0$. This is best seen in Figs. $3 \mathrm{~b}$ and e. Given $m$ and $n$, these angles for each of $P_{m}$ and $P_{n}$ depend on both $m$ and $n$. For large values of $m$ and $n$ the values of $\alpha$ and $\beta$ should be small. For example, setting $\alpha=\beta=10^{\circ}$ is sufficient for the case $m=n=5$ depicted in Fig. 2 . The actual setting is discussed below after the description of the general case $i>5$.

\subsection{Constructing $P_{i}, i \geq 5$}

We construct a polytope such that two facets are visible when looked at from $z=\infty$, and $i-2$ facets are visible when looked at from $z=-\infty$. First, we place 


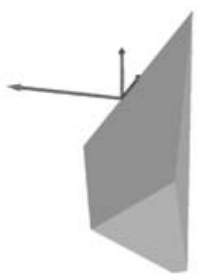

(a)

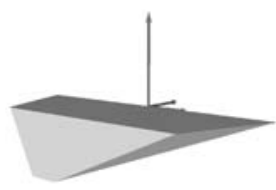

(d)

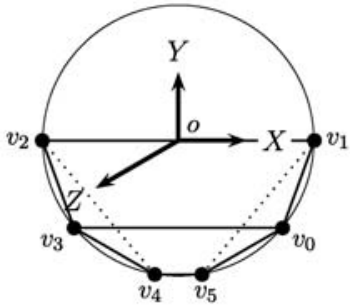

(b)

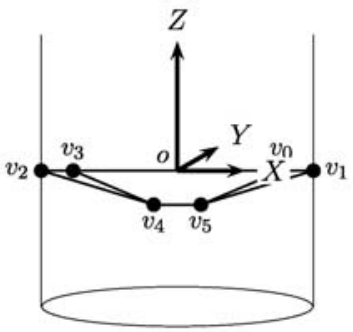

(e)

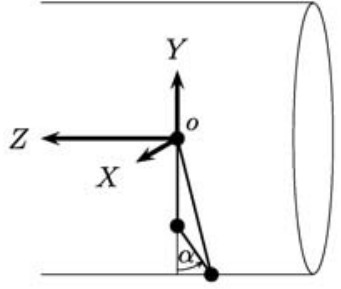

(c)

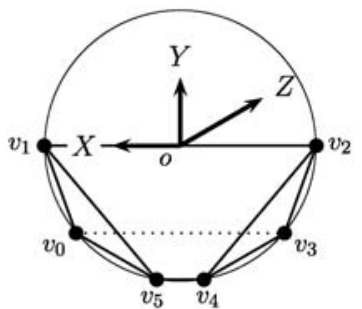

(f)

Fig. 3 Different views of $P_{5}$. a and $\mathbf{d}$ are perspective views, while $\mathbf{b}, \mathbf{c}, \mathbf{e}$, and $\mathbf{f}$ are orthogonal views. Notice that the $Z$ axis is scaled up for clarity

the projection of all vertices onto the plane $z=0$ along the unit circle, and denote the projection of a vertex $v$ as $v^{\prime}$. The projection of the vertices $v_{j_{0}}, v_{j_{1}}, v_{j_{2}}, v_{j_{3}}$, $v_{j_{4}}$, and $v_{j_{5}}$, where $j_{0}=0, j_{1}=\lfloor(i-2) / 2\rfloor, j_{2}=\lfloor(i-2) / 2\rfloor+1, j_{3}=i-2$, $j_{4}=\lfloor(3 i-7) / 2\rfloor$, and $j_{5}=\lfloor(3 i-7) / 2\rfloor+1$, are placed at the same locations as those of the corresponding vertices of $P_{5}$, as depicted below. The projection of the remaining vertices are placed on the arcs $\widehat{v_{j_{5}}^{\prime}, v_{j_{0}}}, \widehat{v_{j_{0}}, v_{j_{1}}}, \widehat{v_{j_{2}}, v_{j_{3}}}$, and $\widehat{v_{j_{3}}, v_{j_{4}}^{\prime}}$ in cyclic order.

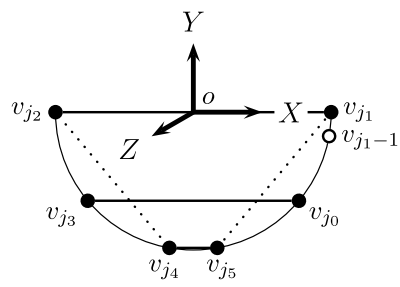

A sketch of $P_{i}, i \geq 5$

The angle $\gamma=\angle v_{j_{0}} o v_{j_{1}-1}$ is another parameter that governs the final configuration of $P_{i}$. Once the placement of the projection of $v_{j_{1}-1}$ is determined, the projections of the vertices $v_{j_{0}+1}, v_{j_{0}+2}, \ldots, v_{j_{1}-2}$ are arbitrarily spread along the open arc $\widehat{v_{j_{0}}, v_{j_{1}}-1}$. The vertex placement along the arc $\widehat{v_{j_{5}}^{\prime}, v_{j_{0}}}$ must be a symmetric reflection of the vertex placement along the arc $\widehat{v_{j_{0}}, v_{j_{1}}}$. This guarantees that all the quadrilateral facets are planar. Similarly, the vertex placement along the arc $\widehat{v_{j_{2}}, v_{j_{3}}}$ is a symmetric reflection of the vertex placement along the arc $\widehat{v_{j_{3}}, v_{j_{4}}^{\prime}}$. For large values of $m$ and $n$, 


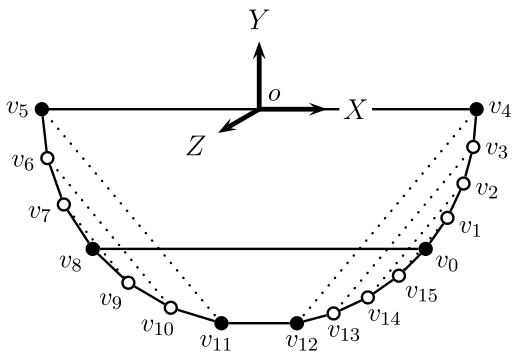

(a)

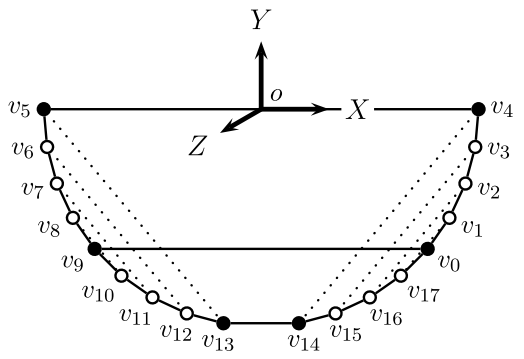

(b)

Fig. 4 a An orthogonal view of $P_{10}$. b An orthogonal view of $P_{11}$

the angle $\gamma$ should be small as explained below, implying that the projection of the vertices are concentrated near $v_{j_{0}}$ and $v_{j_{3}}$ (which lie on the bisectors of $\angle v_{j_{1}} o v_{j_{5}}^{\prime}$ and $\angle v_{j_{2}} o v_{j_{4}}^{\prime}$, respectively). Figure 4 depicts the cases $i=10$ and $i=11$. In these examples, we force a regular placement, which is sufficient in many cases. As in the case of $i=5$, the face $f_{i}^{v}=\overline{v_{0} v_{1} \cdots v_{i-2}}$, represented by the vertex $v_{i}$ of $G_{i}$, lies in the plane $z=0$. The exterior-dihedral angle $\alpha$ at the edge $\overline{v_{j_{0}} v_{j_{3}}}$ is made small so that the vertex $w_{i}$ of $G_{i}$ representing the adjacent face $f_{i}^{w}=\overline{v_{j_{3}} v_{j_{3}+1} \cdots v_{2 i-5} v_{0}}$ is kept in close proximity to $v_{i}$.

Given $m$ and $n$, three parameters per polytope listed below govern the final configurations of $P_{m}$ and $P_{n}$ :

1. The exterior-dihedral angle $\alpha$ at the edge $\overline{v_{j_{0}} v_{j_{3}}}$

2. The angle $\beta=\angle v_{j_{4}}^{\prime} o v_{j_{5}}^{\prime}$

3. The angle $\gamma=\angle v_{j_{0}} o v_{j_{1}-1}$

The settings of these angles must satisfy certain conditions which, in turn, enable all the necessary intersections of edges in the Gaussian map of the Minkowski sum. We denote the face $\overline{v_{j_{5}+1} v_{j_{5}} v_{j_{1}} v_{j_{1}-1}}$ adjacent to $f^{u}$ by $f^{x}$. The vertex $x=G\left(f^{x}\right)$ is the nearest vertex to $u$. The $y$-coordinate of the vertex $w_{n}$ must be greater than the $y$-coordinate of the edge $\overline{x v}_{m}$ at $z=0$ in $P_{m}$ 's coordinate system. Similarly, the $y$-coordinate of the vertex $w_{m}$ must be greater than the $y$-coordinate of the edge $\overline{x v}_{n}$ at $z=0$ in $P_{n}$ 's coordinate system. ${ }^{3}$ This is best seen in Fig. 5c. The values of the $y$-coordinates of $w_{n}$ and $w_{m}$ are simply $\sin \left(\alpha_{n}\right)$ and $\sin \left(\alpha_{m}\right)$, respectively. The value of the $y$-coordinate of the edge $\overline{x v}_{m}$ at $z=0$, however, depends on all the three parameters $\alpha_{m}, \beta_{m}$, and $\gamma_{m}$. Similarly, the $y$-coordinate of the edge $\overline{x v}_{n}$ at $z=0$ in the respective coordinate system depends on $\alpha_{n}, \beta_{n}$, and $\gamma_{n}$. Instead of deriving an expression that directly evaluates these $y$-coordinates, we suggest an iterative procedure that decreases the angles at every iteration until the conditions above are met, and argue that this procedure eventually terminates, because at the limit we are back at the case where $m=n=5$, for which valid settings exist.

\footnotetext{
${ }^{3}$ The rotation of, say $P_{n}$, is performed about the $Y$ axis. Thus, it has no bearing on $y$-coordinates.
} 


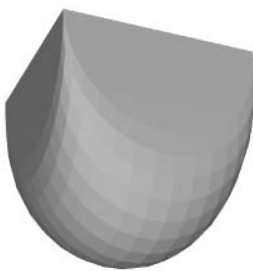

(a)

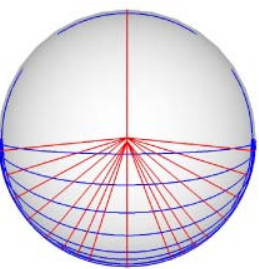

(b)

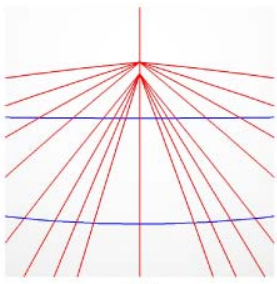

(c)

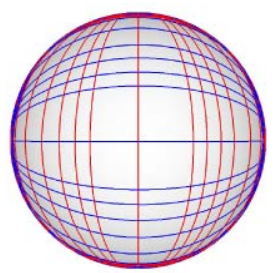

(d)

Fig. 5 a The Minkowski sum $M_{11,11}=P_{11} \oplus P_{11}^{\prime}$, where $P_{11}^{\prime}$ is $P_{11}$ rotated $90^{\circ}$ about the $Y$ axis. b The Gaussian map of $M_{11,11}$ looked at from $z=\infty$. c A scaled up view of the Gaussian map of $M_{11,11}$ looked at from $z=\infty$. d The Gaussian map of $M_{11,11}$ looked at from $y=-\infty$

\subsection{Constructing $P_{4}$}

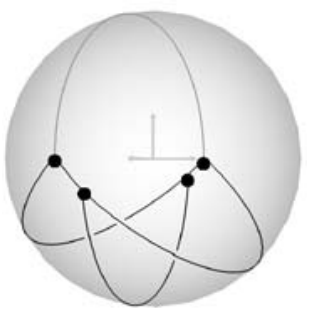

The Gaussian map of $P_{4}$

Recall that $P_{4}$ has 4 facets, 6 edges, and 4 vertices. Therefore, it cannot be constructed according to the prescription provided in the previous section. Applying the same principles though, we place two vertices of $G_{4}$ near the pole $(0,0,-1)$, and two vertices near the opposite pole $(0,0,1)$. One edge, which connects a vertex near one pole to a vertex near the other, lightly shaded in the figure above, is entirely contained in $H^{+}$. The other three edges that connect vertices near opposite poles mostly lie in $H^{-}$. They form a set of $2 i-5=3$ edges, denoted as $E_{4}^{\prime}$. The length of every edge in $E_{4}^{\prime}$ is almost $\pi$. In contrast to the case $i \geq 5$, two out of the three edges in $E_{4}^{\prime}$ cross the plane $y=0$. Namely, small sections of them lie in $H^{+}$. As in the case $i>4$, one edge, the lightly shaded one, is entirely contained in $\mathrm{H}^{+}$.

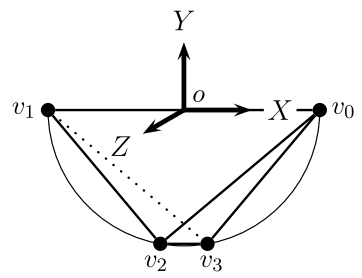

A sketch of $P_{4}$

We construct $P_{4}$, such that the two facets $f^{1}=\overline{v_{0} v_{1} v_{2}}$ and $f^{2}=\overline{v_{0} v_{2} v_{3}}$ are visible when looked at from $z=\infty$, and, when looked at from $z=-\infty$, the remaining two facets $f^{3}=\overline{v_{3} v_{1} v_{0}}$ and $f^{4}=\overline{v_{3} v_{2} v_{1}}$ are visible. As depicted above, the projection 
Fig. 6 The overlay of the Gaussian maps of three tetrahedra rotated about the $Y$ axis $0^{\circ}, 60^{\circ}$, and $120^{\circ}$, respectively

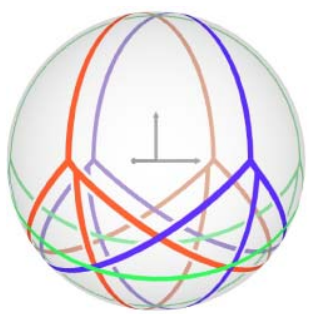

of all four vertices onto the plane $z=0$ lie on the unit circle. The vertices $v_{0}$ and $v_{2}$ lie on the plane $z=0$, and the vertices $v_{1}$ and $v_{3}$ lie in a parallel plane. The distance between the planes is small to form small exterior-dihedral angles at the edges $\overline{v_{0} v_{2}}$ and $\overline{v_{1} v_{3}}$.

As in the general case, two parameters govern the exact placement of $v_{1}, v_{2}$, and $v_{3}$. One is the size of the exterior-dihedral angle at the edge $\overline{v_{0} v_{2}}$. The other parameter is the size of the angle $\angle v_{2} o v_{3}^{\prime}$, where $v_{3}^{\prime}$ is the projection of $v_{3}$ onto the plane $z=0$. The sizes of these angles are determined by the same rationale as in the general case.

This concludes the proof of the lower bound of Theorem 1 for the special case $k=2$.

\section{Maximum Complexity of Minkowski Sums of Many Polytopes}

Let $P_{1}, P_{2}, \ldots, P_{k}$ be a set of $k$ polytopes in $\mathbb{R}^{3}$, such that the number of facets of $P_{i}$ is $m_{i}$ for $i=1,2, \ldots, k$. In this section, we present a tight bound on the number of facets of the Minkowski sum $M=P_{1} \oplus P_{2} \oplus \cdots \oplus P_{k}$, generalizing the arguments presented above for $k=2$. In the following subsections, we establish the lower bound and prove the matching upper bound.

\subsection{The Lower Bound}

Given $k$ positive integers $m_{1}, m_{2}, \ldots, m_{k}$, such that $m_{i} \geq 4$, we describe how to construct $k$ polytopes in $\mathbb{R}^{3}$ with corresponding number of facets, such that the number of facets of their Minkowski sum is exactly $\sum_{1 \leq i<j \leq k}\left(2 m_{i}-5\right)\left(2 m_{j}-5\right)+$ $\sum_{i=1}^{k} m_{i}+\left(\begin{array}{c}k \\ 2\end{array}\right)$. More precisely, given $i$, we describe how to construct a skeleton of a polytope $P_{i}$ with $i$ facets, $3 i-6$ edges, and $2 i-4$ vertices, and prove that the number of facets of the Minkowski sum $M=P_{m_{1}} \oplus P_{m_{2}} \oplus \cdots \oplus P_{m_{k}}$ of the $k$ polytopes properly adjusted and oriented is exactly the expression above. We use the same construction described in Sect. 3.

The number of facets in the Minkowski sum of $P_{i}, i=1,2, \ldots, k$, is maximal when the number of vertices in the overlay of $G_{i}, i=1,2, \ldots, k$, is maximal. This occurs, for example, when $G_{i}$ is rotated $180^{\circ}(i-1) / k$ about the $Y$ axis for $i=$ $1,2, \ldots, k$, as depicted in Fig. 6 for the case of $m_{1}=m_{2}=m_{3}=4$. (Recall that $E_{i}^{\prime}$ refers to the set of edges that span the lowest hemispheres, and its cardinality is smaller than cardinality of $E$ by one.) In this configuration, all the $2 m_{i}-5$ edges in $E_{i}^{\prime}$ intersect all the $2 m_{j}-5$ edges in $E_{j}^{\prime}$, for $1 \leq i<j \leq k$. All intersections occur 
in $H^{-}$. In addition, the edge $\overline{u v}_{m_{i}}$ intersects the edge $\overline{u v}_{m_{j}}$ for $1 \leq i<j \leq k$. These intersection points lie in $\mathrm{H}^{+}$near the pole $(0,1,0)$. Counting all these intersections results with $\sum_{1 \leq i<j \leq k}\left(2 m_{i}-5\right)\left(2 m_{j}-5\right)+\left(\begin{array}{l}k \\ 2\end{array}\right)$. Adding the original vertices of $G\left(P_{i}\right), i=1,2, \ldots, k$, yields the bound asserted in Theorem 1 .

\subsection{The Upper Bound}

We can apply the special case $k=2$ of Theorem 1 to obtain

$$
\begin{aligned}
f\left(m_{1}, m_{2}, \ldots, m_{k}\right) & \leq f\left(m_{1}, f\left(m_{2}, m_{3}, \ldots, m_{k}\right)\right) \\
& \leq 4 m_{1} f\left(m_{2}, m_{3}, \ldots, m_{k}\right)-9 m_{1}-9 f\left(m_{2}, m_{3}, \ldots, m_{k}\right)+26 \\
& \leq 4^{k} \prod_{i=1}^{k} m_{i}+\cdots
\end{aligned}
$$

However, we can apply a technique similar to the one used in Sect. 2 and improve this upper bound, but first we must extend Lemma 3.

Lemma 5 Let $G_{1}, G_{2}, \ldots, G_{k}$ be a set of $k$ Gaussian maps of convex polytopes, and let $G$ be their overlay. Let $f_{i}$ denote the number of faces of $G_{i}$, and let $f$ denote the number of faces of $G$. Then, $f \leq \sum_{1 \leq i<j \leq k} f_{i} f_{j}-(k-2) \sum_{1 \leq i \leq k} f_{i}+$ $(k-1)(k-2)$.

Proof Let us choose two antipodal points on the sphere $\mathbb{S}^{2}$, such that no arc of the overlay is aligned with them. In particular, the points are in the interior of two distinct faces. We consider these two points to be the North pole and South pole of the sphere, and define the direction west to be the clockwise direction when looking from the North pole toward the South pole. We define a western-most corner to be a pair of a face and one of its vertices, which is to the west of all of its other vertices. Apart from the two faces, which contain the poles, any face has a unique western-most corner, since no edge is aligned with the poles, and all faces of any Gaussian map are spherically convex. So for any overlay with $f$ faces, there are $f-2$ such westernmost corners.

The maximal number of faces is attained when the overlay $G$ is non-degenerate. Thus, a vertex of $G$ is either the intersection of two edges of some distinct $G_{i}$ and $G_{j}$, or a vertex of some $G_{i}$. Therefore, a western-most corner for a face of $G$ is either a western-most corner for the overlay of some $G_{i}$ and $G_{j}$, or a western-most corner for some $G_{i}$, in which case it also is a western-most corner for any overlay involving $G_{i}$. The number of western-most corners in the Gaussian map $G_{i}$ is $f_{i}-2$, and the maximal number of western-most corners in the overlay of some $G_{i}$ and $G_{j}$ is $f_{i} f_{j}-2$.

We can therefore write:

$$
f \leq \sum_{1 \leq i<j \leq k}\left(f_{i} f_{j}-2\right)-(k-2) \sum_{i=1}^{k}\left(f_{i}-2\right)+2 .
$$


This corresponds to summing the western-most corners appearing in the overlay of all pairs of Gaussian maps, and subtracting $(k-2)$ times the western-most corners appearing in all original Gaussian maps, since each of them appeared $(k-1)$ times in the first sum. Finally, we have:

$$
\begin{aligned}
& \sum_{1 \leq i<j \leq k}\left(f_{i} f_{j}-2\right)-(k-2) \sum_{i=1}^{k}\left(f_{i}-2\right)+2 \\
& =\sum_{1 \leq i<j \leq k} f_{i} f_{j}-(k-2) \sum_{i=1}^{k} f_{i}+(k-1)(k-2) .
\end{aligned}
$$

Let $P_{1}, P_{2}, \ldots, P_{k}$ be $k$ polytopes in $\mathbb{R}^{3}$ with $m_{1}, m_{2}, \ldots, m_{k}$ facets, respectively. Let $G\left(P_{i}\right)$ denote the Gaussian map of $P_{i}$, and let $v_{i}, e_{i}$, and $f_{i}$ denote the number of vertices, edges, and faces of $G\left(P_{i}\right)$, respectively. Let $v_{x}$ denote the number of intersections of edges of $G\left(P_{i}\right)$ and edges of $G\left(P_{j}\right), i \neq j$ in $G(M)$. Applying the same technique as in Sect. 2, that is, counting the total degrees of vertices in $G(M)$ implies that $\sum_{i=1}^{k} e_{i}+2 v_{x}=e$. Using Euler's formula, we get $\sum_{i=1}^{k} e_{i}+2 v_{x}=$ $f+v-2$. Applying Lemma 5 and respecting $v=\sum_{1 \leq i \leq k} v_{i}+v_{x}$, we can bound $v_{x}$ from above:

$$
v_{x} \leq \sum_{1 \leq i<j \leq k} f_{i} f_{j}-(k-2) \sum_{i=1}^{k} f_{i}+(k-1)(k-2)+\sum_{i=1}^{k}\left(v_{i}-e_{i}\right)-2 .
$$

According to Corollary 4, the maximum number of facets of the Minkowski sum of two polytopes is attained when the number of edges of each summand is maximal. We need to establish a similar property for the general case. Generalizing the derivation procedure in Sect. 2, we introduce $k$ non-negative integers $\ell_{i}, i=1,2, \ldots, k$, such that $e_{i}=3 v_{i}-6-\ell_{i}$ and $f_{i}=2 v_{i}-4-\ell_{i}$. Substituting $e_{i}$ in (2), we get:

$$
\begin{aligned}
v_{x} & \leq \sum_{1 \leq i<j \leq k} f_{i} f_{j}-(k-2) \sum_{i=1}^{k} f_{i}+(k-1)(k-2)+\sum_{i=1}^{k}\left(v_{i}-3 v_{i}+6+\ell_{i}\right)-2 \\
& \leq \sum_{1 \leq i<j \leq k} f_{i} f_{j}-(k-2) \sum_{i=1}^{k} f_{i}-\sum_{i=1}^{k}\left(2 v_{i}-5\right)+\sum_{i=1}^{k} \ell_{i}+k^{2}-2 k .
\end{aligned}
$$

Substituting $f_{i}$ in (3), we get:

$$
\begin{aligned}
v_{x} \leq & \sum_{1 \leq i<j \leq k}\left(2 v_{i}-4-\ell_{i}\right)\left(2 v_{j}-4-\ell_{j}\right)-(k-2) \sum_{i=1}^{k}\left(2 v_{i}-4-\ell_{i}\right) \\
& -\sum_{i=1}^{k}\left(2 v_{i}-5-\ell_{i}\right)+k^{2}-2 k \\
= & \sum_{1 \leq i<j \leq k}\left(2 v_{i}-5\right)\left(2 v_{j}-5\right)+\left(\begin{array}{l}
k \\
2
\end{array}\right)+h\left(\ell_{1}, \ell_{2}, \ldots, \ell_{k}\right)
\end{aligned}
$$


where

$$
\begin{aligned}
h\left(\ell_{1}, \ell_{2}, \ldots, \ell_{k}\right) & =\sum_{1 \leq i<j \leq k}\left(\ell_{i} \ell_{j}-\ell_{j}\left(2 v_{i}-5\right)-\ell_{i}\left(2 v_{j}-5\right)\right) \\
& =\sum_{i=1}^{k} \ell_{i}\left(\sum_{j \neq i}\left(\ell_{j} / 2-\left(2 v_{j}-5\right)\right)\right) .
\end{aligned}
$$

Connectivity of $G\left(P_{i}\right)$ implies that $\ell_{i} \leq 2 v_{i}-5$, which, in turn, implies that $h\left(\ell_{1}, \ell_{2}, \ldots, \ell_{k}\right) \leq 0$. Thus, we have:

$$
v_{x} \leq \sum_{1 \leq i<j \leq k}\left(2 v_{i}-5\right)\left(2 v_{j}-5\right)+\left(\begin{array}{l}
k \\
2
\end{array}\right) .
$$

We conclude that the exact maximum number of facets of the Minkowski sum of $k$ polytopes cannot exceed $\sum_{1 \leq i<j \leq k}\left(2 m_{i}-5\right)\left(2 m_{j}-5\right)+\sum_{1 \leq i \leq k} m_{i}+\left(\begin{array}{l}k \\ 2\end{array}\right)$, which completes the proof of Theorem 1 . For example, the exact maximum number of facets of the Minkowski sum of $k$ tetrahedra is $5 k^{2}-k$.

Acknowledgements We thank Shakhar Smorodinsky for fruitful discussions.

\section{References}

1. Agarwal, P.K., Flato, E., Halperin, D.: Polygon decomposition for efficient construction of Minkowski sums. Comput. Geom. Theory Appl. 21, 39-61 (2002)

2. Aronov, B., Sharir, M.: On translational motion planning of a convex polyhedron in 3-space. SIAM J. Comput. 26(6), 1785-1803 (1997)

3. Basch, J., Guibas, L.J., Ramkumar, G.D., Ramshaw, L.: Polyhedral tracings and their convolution. In: Laumond, J.P., Overmars, M. (eds.) Proceedings of 2nd Workshop on the Algorithmic Foundations of Robotics, pp. 171-184. A.K. Peters, Wellesley (1996)

4. Bekker, H., Roerdink, J.B.T.M.: An efficient algorithm to calculate the Minkowski sum of convex 3D polyhedra. In: Proceedings of International Conference on Computational Science Part I, pp. 619-628. Springer, Berlin (2001)

5. Cameron, S.A., Culley, R.K.: Determining the minimum translational distance between two convex polyhedra. In: Proceedings of IEEE International Conference on Robotics and Automation, pp. 591596 (1986)

6. Fogel, E., Halperin, D.: Exact and efficient construction of Minkowski sums of convex polyhedra with applications. Comput. Aided Des. 39(11), 929-940 (2007)

7. Fukuda, K.: From the zonotope construction to the Minkowski addition of convex polytopes. J. Symb. Comput. 38(4), 1261-1272 (2004)

8. Fukuda, K., Weibel, C.: f-vectors of Minkowski additions of convex polytopes. Discrete Comput. Geom. 37, 503-516 (2007)

9. Ghosh, P.K.: A unified computational framework for Minkowski operations. Comput. Graph. 17(4), 357-378 (1993)

10. Granados, M., Hachenberger, P., Hert, S., Kettner, L., Mehlhorn, K., Seel, M.: Boolean operations on 3D selective Nef complexes: Data structure, algorithms, and implementation. In: Proceedings of 11th Annual European Symposium on Algorithms (ESA). LNCS, vol. 2832, pp. 174-186. Springer, Berlin (2003)

11. Gritzmann, P., Sturmfels, B.: Minkowski addition of polytopes: Computational complexity and applications to Gröbner bases. SIAM J. Discrete Math. 6(2), 246-269 (1993)

12. Guibas, L.J., Ramshaw, L., Stolfi, J.: A kinetic framework for computational geometry. In: Proceedings of 24th Annual IEEE Symposium on the Foundations of Computer Science, pp. 100-111 (1983) 
13. Halperin, D., Kavraki, L., Latombe, J.-C.: Robotics. In: Goodman, J.E., O’Rourke, J. (eds.) Handbook of Discrete and Computational Geometry, 2nd edn., pp. 1065-1093. Chapman \& Hall/CRC, London (2004). Chap. 48

14. Hodgson, C.D., Rivin, I., Smith, W.D.: A characterization of convex hyperbolic polyhedra and of convex polyhedra inscribed in the sphere. Bull. (New Ser.) AMS 27, 246-251 (1992)

15. Kaul, A., Rossignac, J.: Solid-interpolating deformations: Construction and animation of PIPs. In: Proceedings of European Computer Graphics Conference (Eurographics), pp. 493-505 (1991)

16. Lin, M.C., Manocha, D.: Collision and proximity queries. In: Goodman, J.E., O'Rourke, J. (eds.) Handbook of Discrete and Computational Geometry, 2nd edn., pp. 787-807. Chapman \& Hall/CRC, London (2004). Chap. 35

17. Sharir, M.: Algorithmic motion planning. In: Goodman, J.E., O'Rourke, J. (eds.) Handbook of Discrete and Computational Geometry, 2nd edn., pp. 1037-1064. Chapman \& Hall/CRC, London (2004). Chap. 47

18. Weibel, C.: Minkowski sums. http://roso.epfl.ch/cw/poly/public.php 